\title{
Fundamental theorem of hyperbolic geometry without the injectivity assumption
}

\author{
GUOWU YAO \\ Department of Mathematical Sciences, Tsinghua University \\ Beijing, 100084, P.R. China \\ e-mail: gwyao@math.tsinghua.edu.cn
}

\begin{abstract}
Let $\mathbb{H}^{n}$ be the $n$-dimensional hyperbolic space. It is well known that, if $f: \mathbb{H}^{n} \rightarrow$ $\mathbb{H}^{n}$ is a bijection that preserves $r$-dimensional hyperplanes, then $f$ is an isometry. In this paper we make neither injectivity nor $r$-hyperplane preserving assumptions on $f$ and prove the following result:

Suppose that $f: \mathbb{H}^{n} \rightarrow \mathbb{H}^{n}$ is a surjective map and maps an $r$-hyperplane into an $r$-hyperplane, then $f$ is an isometry.

The Euclidean version was obtained by A. Chubarev and I. Pinelis in 1999 among other things. Our proof is essentially different from their and the similar problem arising in the spherical case is open.
\end{abstract}

\section{Introduction}

Let $\hat{\mathbb{R}}^{n}=\mathbb{R}^{n} \cup\{\infty\}$ where $\mathbb{R}^{n}$ is the $n$-dimensional Euclidean space and let $\mathbb{H}^{n}$ be the $n$-dimensional hyperbolic space. A map $f$ of $\hat{\mathbb{R}}^{n}$ to itself is called $r$-sphere preserving if $f$ maps an $r$-dimensional sphere onto an $r$-dimensional sphere. Similarly, a map $f$ of $\mathbb{R}^{n}$ (or $\mathbb{H}^{n}$ ) to $\mathbb{R}^{n}$ (or $\mathbb{H}^{n}$ ) is called $r$-hyperplane preserving if $f$ maps an $r$-dimensional hyperplane onto an $r$-dimensional hyperplane in $\mathbb{R}^{n}$ (or $\mathbb{H}^{n}$ ). In particular, when $r=1$, we call the corresponding map $f$ to be a circle-preserving (line-preserving, geodesic-preserving) map in $\hat{\mathbb{R}}^{n}\left(\mathbb{R}^{n}, \mathbb{H}^{n}\right)$, respectively. In the sequel, we prescribe $n \geq 2$ and $1 \leq r<n$.

The property of a Möbius transformation acting on $\hat{\mathbb{C}}$ is so clear and the relations between Möbius transformation and some of its property have been extensively studied. For examples, Carathéodory first proved that if $f: \hat{\mathbb{C}} \rightarrow \widehat{\mathbb{C}}$ is a circle-preserving bijection, then $f$ is a Möbius transformation (see [4] or [12]); Nehari [11] showed that if $f: \hat{\mathbb{C}} \rightarrow$ $\hat{\mathbb{C}}$ is a non-constant meromorphic function that preserves circles, then $f$ is a Möbius transformation.

Of course, the analogous problem for affine (or isometric) transformations on $\mathbb{R}^{n}$ (or $\left.\mathbb{H}^{n}\right)$ is also concerned. In [7, Jeffers obtained the following extension of Carathéodory's

2000 Mathematics Subject Classification. Primary 37B05, 30C35; Secondary 51F15.

Key words and phrases. Möbius transformation, affine transformation, isometric transformation.

The author was supported by a Foundation for the Author of National Excellent Doctoral Dissertation (Grant No. 200518) and the National Natural Science Foundation of China. 
result to all three cases (for concision, we combine three theorems obtained by him into one).

Theorem A. Suppose that $f: \hat{\mathbb{R}}^{n} \rightarrow \hat{\mathbb{R}}^{n}\left(\mathbb{R}^{n} \rightarrow \mathbb{R}^{n}, \mathbb{H}^{n} \rightarrow \mathbb{H}^{n}\right)$ is a bijection that preserves $r$-dimensional spheres ( $r$-dimensional hyperplanes). Then $f$ is a(n) Möbius (affine, isometric) transformation.

An $r$-sphere preserving map $f$ is called degenerate if its image $f\left(\hat{\mathbb{R}}^{n}\right)$ is an $r$-dimensional sphere; otherwise, $f$ is called non-degenerate. The reader will easily guess the proper definitions for non-degenerate and degenerate maps in the Euclidean and hyperbolic settings. In a recent article [8, B. Li and Y. Wang made neither injectivity nor surjectivity assumptions on $f$ and proved

Theorem B. Suppose that $f: \hat{\mathbb{R}}^{n} \rightarrow \hat{\mathbb{R}}^{n}\left(\mathbb{R}^{n} \rightarrow \mathbb{R}^{n}, \mathbb{H}^{n} \rightarrow \mathbb{H}^{n}\right)$ is a circle-preserving (line-preserving, geodesic-preserving) map. Then $f$ is a(n) Möbius (affine, isometric) transformation if and only if $f$ is non-degenerate.

The existence of degenerate maps was shown in [8, 14. More recently, the author joint with B. Li [9] obtained the following generalization of Theorem B.

Theorem C. Suppose that $f: \hat{\mathbb{R}}^{n} \rightarrow \hat{\mathbb{R}}^{n}\left(\mathbb{R}^{n} \rightarrow \mathbb{R}^{n}, \mathbb{H}^{n} \rightarrow \mathbb{H}^{n}\right)$ is an $r$-sphere preserving ( $r$-hyperplane preserving) map. Then $f$ is a(n) Möbius (affine, isometric) transformation if and only if $f$ is non-degenerate.

In [5], Chubarev and Pinelis showed, among other things, that the injective condition for the Euclidean case $\mathbb{R}^{n}$ in Theorem $\mathrm{A}$ can be removed. Precisely, the following theorem was implied.

Theorem D. Suppose that $f: \mathbb{R}^{n} \rightarrow \mathbb{R}^{n}$ is a surjective map and maps every $r$-dimensional hyperplane into an $r$-dimensional hyperplane. Then $f$ is an affine transformation.

Inspired by Theorem $\mathrm{D}$, the following two conjectures were naturally posed in $[9]$ :

Conjecture 1. Suppose that $f: \hat{\mathbb{R}}^{n} \rightarrow \hat{\mathbb{R}}^{n}$ is a surjective map and maps every $r$-dimensional sphere into an $r$-dimensional sphere. Then $f$ is a Möbius transformation.

and

Conjecture 2. Suppose that $f: \mathbb{H}^{n} \rightarrow \mathbb{H}^{n}$ is a surjective map and maps every $r$-dimensional hyperplane into an $r$-dimensional hyperplane. Then $f$ is an isometric transformation.

The aim of this paper is to prove Conjecture 2 by applying Theorem $\mathrm{C}$ but leave Conjecture 1 open. For completeness, we also give a simple proof of Theorem $\mathrm{D}$ in Section 5.

Other results in the line can be found in $1,12,5,6,10,13$.

Remark 1. Recently, the author [15] proved that Conjecture 1 is true in the case $r=n-1$. 


\section{Some preparations}

This section is devoted to reduce the proof of Conjecture 2 to that of the special case when $r=1$. That is, we only need to prove that,

Theorem 1. Suppose that $f: \mathbb{H}^{n} \rightarrow \mathbb{H}^{n}$ is a surjective map and maps every geodesic into a geodesic. Then $f$ is an isometric transformation.

This reduction clearly depends on the following lemma.

Lemma 1. Suppose (i) there exists some $r$ such that the map $f: \mathbb{H}^{n} \rightarrow \mathbb{H}^{n}$ maps every $r$-dimensional hyperplane into an $r$-dimensional hyperplane, (ii) $f\left(\mathbb{H}^{n}\right)$ is not contained in an $r$-dimensional hyperplane. Then for any given $k$-dimensional hyperplane $\Gamma \subseteq \mathbb{H}^{n}$ $(1 \leq k \leq r), f$ maps $\Gamma$ into a $k$-dimensional hyperplane. In particular, $f$ maps a geodesic into a geodesic.

Throughout our discussion, lower case letters will denote points, upper case letters sets of points, subscripts for like objects, and primes for images under the map $f$. The notable exception to these conventions will be when the image $f(\Lambda)$ of a set $\Lambda$ is not presumed to be $\Lambda^{\prime}$ but we will write $f(\Lambda) \subseteq \Lambda^{\prime}$.

For a nonempty subset $A$ with $\# A \geq 2$ in $\mathbb{H}^{n}$, let $\prod A$ denote the $t$-dimensional hyperplane containing $A$ such that $t$ is the smallest positive integer. It is easy to see that $\prod A$ and $t$ are uniquely determined by the set $A$.

Now, we prove Lemma 1:

If $r=1$, it is a fortiori. Let $r \geq 2$ and $k=r-1$. Embedding $S$ into some $r$-dimensional hyperplane $\Gamma$, we have $\Gamma^{\prime}=f(\Gamma)$ as an $r$-dimensional hyperplane by hypothesis. Since $f\left(\mathbb{H}^{n}\right)$ is not contained in an $r$-dimensional hyperplane, we can find a point $p \in \mathbb{H}^{n} \backslash \Gamma$ such that $p^{\prime}=f(p) \notin \Gamma^{\prime}$.

Letting $\Gamma_{1}=\prod\{S, p\}$, then $\Gamma_{1}$ is an $r$-dimensional hyperplane. Set $\Gamma_{1}^{\prime}=f\left(\Gamma_{1}\right)$. Since

$$
f(S)=f\left(\Gamma \cap \Gamma_{1}\right) \subseteq f(\Gamma) \cap f\left(\Gamma_{1}\right)
$$

and $f(\Gamma) \cap f\left(\Gamma_{1}\right)=\Gamma^{\prime} \cap \Gamma_{1}^{\prime}$ is contained in an $(r-1)$-dimensional hyperplane, the lemma holds for $k=r-1$. It is clear that $f\left(\mathbb{H}^{n}\right)$ is also not contained in an $(r-1)$-dimensional hyperplane. Thus, we can inductively backward prove that if this lemma holds for $k(\geq 2)$, then it does for $k-1$. This lemma then follows.

\section{Proof of Theorem 1}

In this section, we prove Theorem 1. Throughout this section except in Lemma 2 , we assume that $f$ satisfies the conditions of Theorem 1, $l_{x y}$ always denotes the geodesic determined by $x$ and $y$ in $\mathbb{H}^{n}$.

Lemma 2. Suppose $f: \mathbb{H}^{n} \rightarrow \mathbb{H}^{n}$ maps a geodesic into a geodesic. Then $f$ maps an $r$-dimensional hyperplane into an $r$-dimensional hyperplane for $1 \leq r \leq n-1$.

Proof. We use induction. Let $n \geq 3$ and assume that $f$ maps an $r$-dimensional hyperplane into an $r$-dimensional hyperplane for some $r \in[1, n-2]$. We need to show that $f$ maps an $(r+1)$-dimensional hyperplane into an $(r+1)$-dimensional hyperplane. 
Suppose not. Then there exists an $(r+1)$-dimensional hyperplane $S$ such that $\prod f(S)$ has dimension $d \geq r+2$. Therefore, there exist $r+3$ points $\left\{p_{1}^{\prime}, p_{2}^{\prime}, \cdots, p_{r+3}^{\prime}\right\}$ in $f(S)$ such that no $(r+1)$-dimensional hyperplane contains them and the hyperplane $\prod K^{\prime}$ spanned by $K^{\prime}=\left\{p_{1}^{\prime}, p_{2}^{\prime}, \cdots, p_{r+3}^{\prime}\right\}$ has dimension $r+2$. On the other hand, there exist $r+3$ distinct points $\left\{p_{1}, p_{2}, \cdots, p_{r+3}\right\}$ in $S$ such that $f\left(p_{i}\right)=p_{i}^{\prime}(i=1,2, \cdots, r+3)$.

It is clear that no $r$-dimensional hyperplane contains more than $r+2$ points of $\left\{p_{1}, p_{2},-\right.$ $\left.\cdots, p_{r+3}\right\}$ by the inductive assumption. Therefore, every $r+1$ points of $\left\{p_{1}, p_{2}, \cdots, p_{r+2}\right\}$ can span a unique $r$-dimensional hyperplane and these $r+2$ spanned hyperplanes divide the $(r+1)$-dimensional hyperplane $S$ into $2^{r+2}-1$ disjoint parts. The point $p_{r+3}$ is located inside some part.

Observe that $\left\{p_{1}, p_{2}, \cdots, p_{r+2}\right\}$ frames an $(r+1)$-simplex in $S$. Anyway there exists at least a point of $\left\{p_{1}, p_{2}, \cdots, p_{r+2}\right\}$, say $p_{1}$, such that the geodesic $l_{p_{1} p_{r+3}}$ crosses the $r$-dimensional hyperplane $\Lambda=\prod\left\{p_{2}, p_{3}, \cdots, p_{r+2}\right\}$. Letting $q=l_{p_{1} p_{r+3}} \cap \Lambda$, then $q \neq$ $p_{1}$ and $q^{\prime}=f(q) \in \prod\left\{p_{2}^{\prime}, p_{3}^{\prime}, \cdots, p_{r+2}^{\prime}\right\}$ by the inductive assumption. Thus, $p_{r+3}^{\prime}=$ $f\left(p_{r+3}\right) \in f\left(l_{q p_{1}}\right)$ which shows that $p_{r+3}^{\prime} \in \prod\left\{p_{1}^{\prime}, p_{2}^{\prime}, \cdots, p_{r+2}^{\prime}\right\}$ since $f\left(l_{q p_{1}}\right) \subseteq l_{q^{\prime} p_{1}^{\prime}}$. This further indicates that $\left\{p_{1}^{\prime}, p_{2}^{\prime}, \cdots, p_{r+3}^{\prime}\right\}$ is contained in the $(r+1)$-dimensional hyperplane $\prod\left\{p_{1}^{\prime}, p_{2}^{\prime}, \cdots, p_{r+2}^{\prime}\right\}$, a contradiction. The inductive proof is completed.

Remark 2. Lemma 2 can be regarded as a converse of Lemma 1. We have an essential difficulty in obtaining its spherical version which is also the only bug to solve Conjecture 1 while we prove Conjecture 1 when $r=n-1$ in [15. In other words, the answer to the following problem is crucial to the solution of Conjecture 1 .

Problem 1. Suppose that $f: \hat{\mathbb{R}}^{n} \rightarrow \hat{\mathbb{R}}^{n}(n \geq 3)$ is a surjective map and maps a circle into a circle. Can we say that $f$ maps an $(n-1)$-dimensional sphere into an $(n-$ 1)-dimensional sphere?

Lemma 3. Suppose $D$ is a domain in $\mathbb{H}^{n}$. If $f(D)$ is contained in an $(n-1)$-dimensional hyperplane, then $f$ is constant on $D$.

Proof. Suppose not. Then $f(D)$ is contained in an $(n-1)$-dimensional hyperplane, say $\Gamma^{\prime} \subseteq \mathbb{H}^{n}$, and $f(D)$ contains at least two points. Let $S=\left\{w \in \mathbb{H}^{n}: f(w) \in \Gamma^{\prime}\right\}$. Obviously, $D \subseteq S, f(S) \subseteq \Gamma^{\prime}$ and $S \neq \mathbb{H}^{n}$.

Claim 1. $S$ is path-connected.

We may choose two points $p, q$ in $S$ such that $f(p) \neq f(q)$ since $f(S)=\Gamma^{\prime}$. Now, for any other point $w \in S$, it is no harm to assume that $f(w) \neq f(q)$. Thus, the geodesic $l_{w q} \subseteq S$ since $f\left(l_{w q}\right) \subseteq \Gamma^{\prime}$ which implies that $S$ is path-connected.

Claim 2. $\mathbb{H}^{n}-S$ contains no interior points.

Suppose to the contrary. Let $p$ be an interior point of $\mathbb{H}^{n}-S$ and $P$ be the largest connected open set in $\mathbb{H}^{n} \backslash S$ such that $p \in P$. Whence, every set $f\left(l_{p q} \cap D\right)$ is a singleton since otherwise $p^{\prime} \in \Gamma^{\prime}$, where the geodesic $l_{p q}$ passes through $p$ and a point $q \in D$.

Since $f(D)$ contains at least two points, there are two points, say $u$ and $v$, such that the geodesics $l_{p^{\prime} u^{\prime}}$ and $l_{p^{\prime} v^{\prime}}$ are distinct. Therefore, there exists at least a point $x$ in $D$ and a sequence of points $\left\{x_{n}\right\}_{n=1}^{\infty}$ in $D$ such that

$$
\lim _{n \rightarrow \infty} x_{n}=x \text { and } l_{p^{\prime} x_{n}^{\prime}} \neq l_{p^{\prime} x^{\prime}}, \forall n .
$$


Observe that $f\left(l_{p x_{n}} \cap D\right)=x_{n}^{\prime}$ and $f\left(l_{p x} \cap D\right)=x^{\prime}$ and $x_{n}^{\prime} \neq x^{\prime}$ for all $n$. We then may choose sufficiently large integer $m$ and a point $y_{m}$ in $l_{p x_{m}} \cap D$ such that the geodesic $l_{x y_{m}}$ through $x$ and $y_{m}$ crosses the domain $P$. Recalling that $f(x)=x^{\prime}$ and $f\left(y_{m}\right)=x_{m}^{\prime}$, we can find a point $z \in l_{x y_{m}} \cap P$ such that $f(z) \in f\left(l_{x y_{m}}\right) \subseteq \Gamma^{\prime}$. This indicates that $z \in S$, a contradiction.

We continue to derive a new contradiction from the above two Claims as follows.

Let $\Lambda^{\prime} \subseteq \mathbb{H}^{n} \backslash \Gamma^{\prime}$ be an $(n-1)$-dimensional hyperplane. Choose $n$ points $\left\{p_{1}^{\prime}, p_{2}^{\prime}, \cdots, p_{n}^{\prime}\right\}$ in $\Lambda^{\prime}$ such that these $n$ points are not contained in an $(n-2)$ - dimensional hyperplane (when $n=2$, such choice is trivial). There exist $n$ distinct points $\left\{p_{1}, p_{2}, \cdots, p_{n}\right\}$ in $\mathbb{H}^{n}$ such that $f\left(p_{i}\right)=p_{i}^{\prime}(i=1,2, \cdots, n)$. Let $\Lambda=\prod\left\{p_{1}, p_{2}, \cdots, p_{n}\right\}$ be the hyperplane spanned by $\left\{p_{1}, p_{2}, \cdots, p_{n}\right\}$. It is easy to deduce from Lemma 2 that the dimension $\operatorname{dim}(\Lambda)$ of $\Lambda$ is just $n-1$ and $f(\Lambda) \subseteq \Lambda^{\prime}$. Notice that $\Lambda$ divides $\mathbb{H}^{n}$ into two disjoint domains. Necessarily, $\Lambda \cap S \neq \emptyset$ by Claims 1 and 2. Thus, we have $f(\Lambda \cap S) \neq \emptyset$ which contradicts that $f(\Lambda) \cap f(S) \subseteq \Lambda^{\prime} \cap \Gamma^{\prime}=\emptyset$. This completes the proof of Lemma 3 ,

Lemma 4. Suppose $D$ is a domain in $\mathbb{H}^{n}$. Then $f(D)$ cannot be contained in an $(n-$ 1)-dimensional hyperplane in $\mathbb{H}^{n}$.

Proof. Suppose not. Then by Lemma 3, $f$ is constant on $D$, in other words, $f$ maps $D$ to a point, say $p^{\prime} \in \mathbb{H}^{n}$. Let $\mathscr{D}$ be the largest connected open set of $\mathbb{H}^{n}$ such that $f(\mathscr{D})=\left\{p^{\prime}\right\}$. Set $S=\left\{w \in \mathbb{H}^{n}: f(w)=p^{\prime}\right\}$. Obviously, $D \subseteq \mathscr{D} \subseteq S$ and $S \neq \mathbb{H}^{n}$.

Claim. $\mathbb{H}^{n}-S$ contains interior points.

We may choose two $(n-1)$-dimensional hyperplanes $\Phi^{\prime}$ and $\Psi^{\prime}$ in $\mathbb{H}^{n} \backslash\left\{p^{\prime}\right\}$ such that, (i) $\Phi^{\prime} \cap \Psi^{\prime}=\emptyset$ and (ii) the convex set $K^{\prime}=\left\{z^{\prime} \in \mathbb{H}^{n}: \exists x^{\prime} \in \Phi^{\prime}, y^{\prime} \in \Psi^{\prime}\right.$, s.t. $\left.z^{\prime} \in l_{x^{\prime} y^{\prime}}\right\}$ does not contain $p^{\prime}$. By virtue of Lemma 2, one can find two hyperplanes $\Phi$ and $\Psi$ in $\mathbb{H}^{n}$ such that $f(\Phi) \subseteq \Phi^{\prime}, f(\Psi) \subseteq \Psi^{\prime}$ and $\operatorname{dim}(\Phi)=\operatorname{dim}(\Psi)=n-1$. It is evident that $\Phi \cap \Psi=\emptyset$. It is also clear that the convex set $K=\left\{z \in \mathbb{H}^{n}: \exists x \in \Phi, y \in \Psi\right.$, s.t. $\left.z \in l_{x y}\right\}$ contains interior points and $f(K) \subseteq K^{\prime}$. This claim follows immediately.

Now, in virtue of the above Claim, it is easy to choose an interior point $q$ of $\mathbb{H}^{n} \backslash S$ and a point $b$ on the boundary of $\mathscr{D}$ such that geodesics through $p$ and points of $\mathscr{D}$ contain a neighborhood $N$ of $b$. Noticing that all such geodesics are mapped into $l_{q^{\prime} p^{\prime}}$, we have $N \subseteq S$ by Lemma 3 . This contradiction establishes this lemma.

Lemma 5. $f$ maps every $(n-1)$-dimensional hyperplane in $\mathbb{H}^{n}$ onto an $(n-1)$-dimensional hyperplane in $\mathbb{H}^{n}$, i.e., $f$ is $(n-1)$-hyperplane preserving.

Proof. Given an $(n-1)$-dimensional hyperplane $S$ in $\mathbb{H}^{n}$, by Lemma 2, there exists an $(n-1)$-dimensional hyperplane $S^{\prime} \supset f(S)$. We now show that $S^{\prime}=f(S)$. Suppose not, then there should exist some point $a^{\prime} \in S^{\prime} \backslash f(S)$. Let $a \in \mathbb{H}^{n} \backslash S$ be an inverse image of $a^{\prime}$ under $f$. The collection of geodesics through $a$ and points of $S$ covers a domain in $\mathbb{H}^{n}$ which is mapped into the $(n-1)$-dimensional hyperplane $S^{\prime}$. It derives a desired contradiction from Lemma 4. Thus, we prove that $f$ is $(n-1)$-hyperplane preserving.

Finally, the proof of Theorem 1 is concluded by Lemma 5 and Theorem C(let $r=n-1)$. 


\section{A simple proof of Theorem D}

By the foregoing reasoning, the proof of Theorem $\mathrm{D}$ reduces to that of the following theorem.

Theorem 2. Suppose that $f: \mathbb{R}^{n} \rightarrow \mathbb{R}^{n}$ is a surjective map and maps every line into a line. Then $f$ is an affine transformation.

Proof. For one thing, it is easy to prove that $f$ maps an $(n-1)$-dimensional hyperplane into an $(n-1)$-dimensional hyperplane as proving Lemma 2 ,

We claim that $f$ also maps an $(n-1)$-dimensional hyperplane onto an $(n-1)$-dimensional hyperplane. Suppose to the contrary. Then there exists an $(n-1)$-dimensional hyperplane $\Gamma$ in $\mathbb{R}^{n}$ such that $f(\Gamma)$ is contained in an $(n-1)$-dimensional hyperplane $\Gamma^{\prime}$ and $\Gamma^{\prime} \backslash f(\Gamma) \neq \emptyset$.

Let $p^{\prime} \in \Gamma^{\prime} \backslash f(\Gamma)$ and $p$ an inverse image of $p^{\prime}$. Observe that lines through $p$ and points in $\mathbb{R}^{n} \backslash\{p\}$ either cross $\Gamma$ or parallel $\Gamma$, and the formers are mapped into $\Gamma^{\prime}$ and the latters are contained in an $(n-1)$-dimensional hyperplane parallel to $\Gamma$ and hence are mapped into an $(n-1)$-dimensional hyperplane, say $\Lambda^{\prime}$. Thus, $\mathbb{R}^{n}$ is mapped into the union of $\Gamma^{\prime}$ and $\Lambda^{\prime}$, a contradiction.

The proof of Theorem 2 is completed by applying Theorem C,

\section{Concluding remarks}

All results mentioned in this paper belong to a young and active geometrical discipline called "characterizations of geometrical mappings under mild hypotheses". The discipline started around 1950 with fundamental theorems of A. D. Alexandrov on spacetime transformations and causal automorphisms (see [3]). Throughout the conditions in these theorems, for examples, Theorems $A \sim D$ and our main result, surjectivity, injectivity and non-degenerate play inevitable roles. In our result, we remove the injectivity assumption but non-degenerate one is satisfied automatically. In a future paper [16], the author even further replaces the surjectivity assumption with the condition that every $r$-dimensional hyperplane contains at least $r+1$ image points. One may ask, what situation will be if the surjectivity assumption on $f$ replaced by the injectivity one?

Actually, we can say nothing on $f$ because there exists a so-called degenerate map $f: \mathbb{H}^{n} \rightarrow \mathbb{H}^{n}$ such that $f$ maps $\mathbb{H}^{n}$ one-to-one into some $\tilde{r}$-dimensional hyperplane. So, we need another restriction on $f$ to guarantee that it is an automorphism. Naturally, "non-degenerate" is the first candidate. Maybe one expects a theorem as follows:

If $f: \mathbb{H}^{n} \rightarrow \mathbb{H}^{n}$ is an injective map and maps an $r$-dimensional hyperplane into an $r$-dimensional hyperplane and if $f$ is non-degenerate, i.e., $f\left(\mathbb{H}^{n}\right)$ cannot be contained in an $r$-dimensional hyperplane, then $f$ is an isometry.

Unfortunately, recently in oral communication, a counterexample was given by Li Baokui. We interpret it here.

Counterexample: For convenience, let $n=2$ and use the semisphere $S$ in $\mathbb{R}^{3}$ as the model of $\mathbb{H}^{2}$, namely,

$$
S=\left\{(x, y, z) \in \mathbb{R}^{3}: x^{2}+y^{2}+z^{2}=1, z>0\right\} .
$$


Then, all geodesics in $S$ are these semicircles perpendicular to the $X O Y$-plane. Project $S$ onto the unit disk $D$ in $\mathbb{R}^{2}$ :

$$
D=\left\{(x, y) \in \mathbb{R}^{2}: x^{2}+y^{2}<1\right\} .
$$

Say, let $P$ denote the projection:

$$
P(x, y, z)=(x, y) .
$$

It is easy to see that the images of geodesics in $S$ under $P$ are these segments with ends at the boundary of $D$. Let $A$ be an affine transformation in $\mathbb{R}^{2}$ with the form:

$$
A(x, y)=(a x, b y), a, b \in(0,1)
$$

Thus, the composition map $f=P^{-1} \circ A \circ P$ maps $S$ into $S$. It is evident that $f$ is injective and non-degenerate. It maps a geodesic in $S$ into a geodesic and the action of $f$ on geodesics is shortening them and hence $f$ is not an isometry.

Although the exceptional phenomenon occurs in $\mathbb{H}^{n}$, we cannot find such counterexample in $\hat{\mathbb{R}}^{n}$ or $\mathbb{R}^{n}$ so far. Whence, we end remarks with two open problems.

Problem 2. Suppose $f: \hat{\mathbb{R}}^{n} \rightarrow \hat{\mathbb{R}}^{n}$ is an injective map and maps an $r$-dimensional sphere into an r-dimensional sphere. Can we say that $f$ is a Möbius transformation if $f\left(\hat{\mathbb{R}}^{n}\right)$ cannot be contained in an $r$-dimensional sphere?

Problem 3. Suppose $f: \mathbb{R}^{n} \rightarrow \mathbb{R}^{n}$ is an injective map and maps an $r$-dimensional hyperplane into an $r$-dimensional hyperplane. Can we say that $f$ is an affine transformation if $f\left(\mathbb{R}^{n}\right)$ cannot be contained in an $r$-dimensional hyperplane?

Acknowledgements. The author would like to express gratitude to the referee for his valuable comments. Thanks are also due to Professor Xiantao Wang for some idea of this paper inspired by helpful communication with him.

\section{REFERENCES}

[1] J. Aczél and M. A. McKiernan, On the characterization of hyperplane projective and complex Möbius-transformation, Math. Nachr. 33 (1967), 315-337.

[2] A. F. Beardon and D. Minda, Sphere-preserving maps in inversive geometry, Proc. Amer. Math. Soc. 130 (2001), 987-998.

[3] W. Benz, Classical Geometries in Modern Contexts [Geometry of Real Inner Product Spaces], Birkhäuser, Basel-Boston-Berlin, 2005.

[4] C. Carathéodory, The most general transformations of plane regions which transform circles into circles, Bull. Amer. Math. Soc. 43 (1937), 573-579.

[5] A. Chubarev and I. Pinelis, Fundamental theorem of geometry without the 1-to-1 assumption, Proc. Amer. Math. Soc. 127 (1999), 2735-2744. 
[6] J. Gibbons and C. Webb, Circle-preserving functions of spheres, Trans. Amer. Math. Soc. 248 (1979), 67-83.

[7] J. Jeffers, Lost theorems of geometry, Amer. Math. Monthly, 107 (2000), 800-812.

[8] B. Li and Y. Wang, Transformations and non-degenerate maps, Sci. China Ser. A, Mathematics, Vol. 48 Supp. (2005), 195-205.

[9] B. K. Li and G. W. Yao, On characterizations of sphere-preserving maps, Math. Proc. Cambridge Philos. Soc. to appear, doi: 10.1017/S0305004109002291, 2009.

[10] R. Löwen, A local "Fundamental Theorem" for classical topological projective spaces, Arch. Math. 38 (1982), 286-288.

[11] Z. Nehari, Conformal Mapping, New York, McGraw-Hill, 1952.

[12] H. Schwerdtfeger, Geometry of Complex Numbers, Mathematical expositions series, no. 13, Univ. of Toronto Press, Toronto, 1962.

[13] G. Steinke, An extension property for classical topological Benz planes, Arch. Math. 41 (1983), 190-192.

[14] G. W. Yao, On existence of degenerate circle-preserving maps, J. Math. Anal. Appl. 334 (2007), 950-953.

[15] G. W. Yao, Transformations of spheres without the injectivity assumption, preprint, 2008.

[16] G. W. Yao, Fundamental theorem of geometry with the injectivity and surjectivity assumptions absent, in preparation. 